OPEN ACCESS

Edited by:

Sree Bhushan Raju,

Nizam's Institute of Medical

Sciences, India

Reviewed by:

Arunkumar Subbiah,

All India Institute of Medical

Sciences, India

Shougang Zhuang,

Brown University, United States

*Correspondence:

Pei Wang

wpei@zzu.edu.cn

Specialty section:

This article was submitted to Nephrology,

a section of the journa

Frontiers in Medicine

Received: 29 October 2021 Accepted: 02 February 2022

Published: 07 March 2022

Citation:

Zhou F, Yao L, LuX, Li Y, Han X and Wang $P$ (2022) Therapeutic Targeting

of GSK3ß-Regulated Nrf2 and NFKB

Signaling Pathways by Salvianolic Acid A Ameliorates Peritoneal Fibrosis.

Front. Med. 9:804899.

doi: 10.3389/fmed.2022.804899

\section{Therapeutic Targeting of} GSK3 $\beta$-Regulated Nrf2 and NFKB Signaling Pathways by Salvianolic Acid A Ameliorates Peritoneal Fibrosis

\author{
Fan Zhou ${ }^{1,2}$, Lan Yao ${ }^{3}$, Xiaoqing $\mathrm{Lu}^{3}$, Yubao $\mathrm{Li}^{3}$, Xingmin $\mathrm{Han}^{1,2}$ and Pei Wang ${ }^{3 *}$ \\ ${ }^{1}$ Department of Nuclear Medicine, The First Affiliated Hospital of Zhengzhou University, Zhengzhou, China, ${ }^{2}$ Henan Medical \\ Key Laboratory of Molecular Imaging, Zhengzhou, China, ${ }^{3}$ Blood Purification Center, Institute of Nephrology, The First \\ Affiliated Hospital of Zhengzhou University, Zhengzhou, China
}

Peritoneal fibrosis is a devastating complication in patients undergoing peritoneal dialysis, with no definite therapy yet available. Salvia miltiorrhiza and its major active component Salvianolic acid A (Sal A) have demonstrated a beneficial effect in myriad diseases. However, their effect on peritoneal fibrosis is unknown. In murine models of peritoneal dialysis, daily Sal A treatment substantially improved the peritoneal dialysis fluid (PDF) elicited peritoneal fibrosis, marked by thickening of the submesothelial compact zone, accumulation of extracellular matrix and increased expression of vimentin and PAI-1, concomitant with attenuation of GSK3 $\beta$ hyperactivity. This coincided with diminished nitrotyrosine in peritoneal tissues and increased Nrf2 nuclear translocation, entailing a lessened oxidative injury and reinforced Nrf2 antioxidant response. Meanwhile, inflammatory infiltration and maladaptive angiogenesis in peritoneal tissues provoked by PDF injury were also mitigated by Sal $A$, associated with a suppressed $N F_{K} B$ activation. Mechanistically, ectopic expression of the constitutively active GSK3 $\beta$ blunted the NFkB-suppressing and Nrf2-activating efficacy of Sal A in peritoneal mesothelial cells exposed to hypertonic dextrose, suggesting that GSK3 $\beta$ inhibition mediates the protective effect of Sal A. Collectively, our findings may open the avenue for developing a novel therapy based on Sal A for preventing peritoneal fibrosis in peritoneal dialysis.

Keywords: peritoneal dialysis, epithelial cells, oxidative stress, inflammation, fibrosis, herb, Salvia miltiorrhiza

\section{INTRODUCTION}

Peritoneal dialysis is one of the very few renal replacement therapeutic modalities available to patients with end-stage renal failure or uremia (1). The efficacy of peritoneal dialysis is highly dependent on the functionality of the peritoneal membrane that lines over the abdominal cavity of the peritoneum and serves as a semipermeable membrane, through which body water and metabolic wastes passes into the dialysis fluid until equilibrium (2). The peritoneal membrane is comprised mainly by the peritoneal mesothelial cells, which may dedifferentiate and acquire profibrogenic phenotypes upon long term exposure to a number of injurious factors, including sustained hypertonicity of peritoneal dialysis solutions, uremic toxins, peritonitis, infection and 
inflammatory mediators, ultimately culminating in peritoneal fibrosis and peritoneal membrane ultrafiltration dysfunction, with no definite therapy yet available $(1,2)$. In order to sustain a critical life-line for uremic patients, it is imperative to identify novel therapeutic targets and develop new treatments for preventing peritoneal fibrosis.

Akin to fibrosis in other organ systems (3), peritoneal fibrosis is a complex yet well-orchestrated pathological process and involves oxidative stress, inflammation, maladaptive angiogenesis and other events (1). A number of cellular signaling pathways have been implicated in this process. Among these, the nuclear factor-erythroid factor 2-related factor 2 (Nrf2) antioxidant response has been regarded as the master mechanism for self-defense against oxidative injury (4). In addition, the $\mathrm{NF \kappa B}$ pathway plays a key role in inflammatory response and maladaptive angiogenic endothelial proliferation (5). Recent studies demonstrate that GSK3 $\beta$ is situated at the nexus of the Nrf2 and NFKB pathways and serves as a crucial regulator of the above pathological events $(6,7)$. Indeed, burgeoning evidence has implicated GSK3 $\beta$ in the fibrogenesis of diverse tissues $(8,9)$, including peritoneal mesothelial cells $(10)$. As such, therapeutic targeting of GSK3 $\beta$ is likely a promising strategy to treat peritoneal fibrosis.

Salvia miltiorrhiza, also known as red sage, is an herb widely used in traditional Chinese medicine to treat cardiovascular and cerebrovascular disease $(11,12)$. A growing body of evidence recently suggests that $S$. miltiorrhiza is likely to confer a multipronged beneficial effect in various organ systems via a potent antioxidant and anti-inflammatory mechanism (11). The main effective molecules are still uncertain, but there is evidence suggesting that $S$. miltiorrhiza extractants contain high amounts of Salvianolic acid A (Sal A), a rosmarinic acid derivative and a member of the class of 1-benzofurans (Figure 1) and an antioxidant, anti-inflammatory, hypoglycemic, hepatoprotective, neuroprotective, cardioprotective agent (11). Mechanistically, Sal $\mathrm{A}$ is able to perturb a number of cell signaling pathways related to fibrogenesis, such as GSK3 $\beta(13,14)$. Recent studies demonstrate a potent anti-fibrotic effect of Salvianolic acid A in multiple organs (15), including the kidney (13), liver (16), and lung (17). However, the effect of $S$. miltiorrhiza or its effective component Sal A on peritoneal fibrosis has not been studied before. This study aimed to examine the possible effect of Sal A on peritoneal fibrosis in vivo in murine models of peritoneal dialysis and in vitro in cultured peritoneal mesothelial cells.

\section{MATERIALS AND METHODS}

\section{Animal Study Design}

Male B6 mice (8-10-weeks old) were used in this study. The mouse model of peritoneal fibrosis was established by daily intraperitoneal injection of $3 \mathrm{ml}$ of $4.25 \%$ dextrose peritoneal dialysis fluid (PDF, Baxter HealthCare, Deerfield, IL, USA) as previously described (18). Mice treated with the same volume of PBS served as normal control. PDF or PBS-treated mice were randomized to receive daily intraperitoneal injection of vehicle or Sal A (60 mg/kg, Sigma, MO, USA) according to the dose used before (19). All animals were euthanized on week 7 and peritoneal tissues including the anterior abdominal wall were collected for further examination. All experimental procedures were approved by the Ethics Committee at the First Affiliated Hospital of Zhengzhou University.

\section{Cell Cultures}

Primary mouse peritoneal mesothelial cells (PMC) were isolated by enzymatic digestion of the inner surface of the peritoneum and then cultured in culture medium containing DMEM with $20 \%$ FBS as described previously (20). In brief, immediately after euthanasia, $10 \mathrm{ml}$ of $0.25 \%$ tripsin solution was injected into the peritoneum of mice. After $10 \mathrm{~min}$, the trypsin solutions were collected with needle and syringe and the peritoneal cavities were rinsed with culture medium, which was collected and transferred together with the trypsin solutions to a centrifuge tube and centrifuged. The cell pellet was collected and re-suspended in culture medium. The cells were characterized to possess typical phenotypes of mesothelial cells, including cytokeratin and calretinin with negligible contamination by leukocytes. Cells were subjected to Lipofectamine-transient transfection with the empty vector or the vector encoding the hemagglutinin (HA)conjugated constitutively-active mutant of GSK3 $\beta$ as previously elaborated (21). $24 \mathrm{~h}$ after transfection, cells were then pretreated with Sal A $(50 \mu \mathrm{M})$ as reported before (22) or vehicle for $30 \mathrm{~min}$ prior to treatment with $4.25 \%$ dextrose or equal volume of PBS for $48 \mathrm{~h}$.

\section{Western Immunoblot Analysis}

Peritoneal tissues or collected cells were lysed in radioimmunoprecipitation assay buffer supplemented with protease inhibitors. NE-PER kit (Thermo Scientific, Rockford, Illinois, USA) was used to prepare the nuclear fractions according to the manufacturer's instruction. Protein samples were processed for immunoblot analysis as previously described (23). The antibodies against fibronectin, vimentin, PAI-1, nitrotyrosine, Histone $\mathrm{H} 3$, and $\beta$-actin were purchased from Santa Cruz Biotechnology (CA, USA). The antibodies against

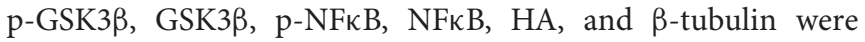
purchased from Cell Signaling Technology (MA, USA). The antibody against Nrf2 was acquired from Abcam (CA, USA).

\section{ELISA Analyses}

Peritoneal lysates were subjected to Enzyme-linked immunosorbent assay (ELISA) for measuring the expression levels of TNF- $\alpha$, IL-1 $\beta$, MCP-1 protein in accordance with the manufacturer's instructions (R\&D Systems, MN, USA).

\section{Histology and Immunostaining}

Formalin-fixed paraffin-embedded peritoneal tissues were processed for sections and following subjected to immunofluorescence staining or Masson's trichrome staining to assess histological changes and peritoneum thickness. The peritoneum sections were deparaffinized and rehydrated, heated in citrate buffer by microwave, quenched with $3 \% \mathrm{H}_{2} \mathrm{O}_{2}$, blocked by $5 \% \mathrm{BSA}$, and incubated with the primary antibodies against the following molecules: CD45 (Santa Cruz Biotechnology), CD68 (Abcam), CD31 (Abcam), vascular endothelial growth 
A

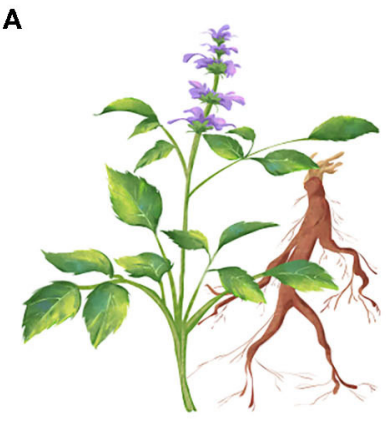

B

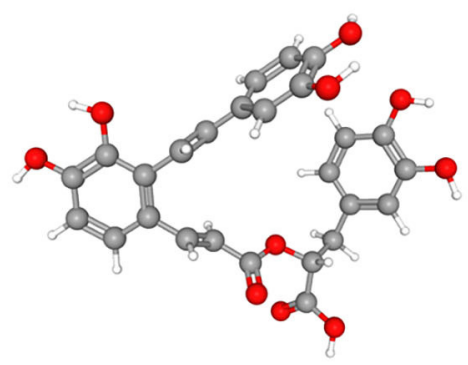

C

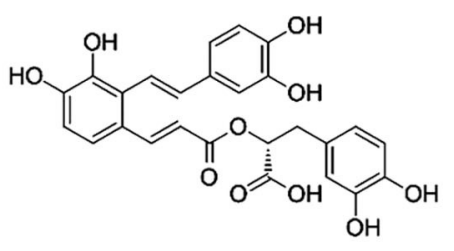

FIGURE 1 | Salvia miltiorrhiza and the chemical structure of its key active component Salvianolic acid A (Sal A). (A) Illustration of Salvia miltiorrhiza and the medicinal root; (B) As the most abundant water-soluble active component extracted from Salvia miltiorrhiza, Sal A is a rosmarinic acid derivative and a member of the class of 1-benzofurans. The 3-D view of the conformer of Sal A; (C) The 2-D view of the chemical structure of Sal A.

factor (VEGF, Santa Cruz Biotechnology), nitrotyrosine (Santa Cruz Biotechnology), Nrf2 (Abcam), followed by staining with Alexa Fluor-conjugated secondary antibody (Life Technologies, Carlsbad, CA, USA). Finally, sections were mounted with mounting medium with 4',6-diamidino-2-phenylindole (DAPI, Abcam), and visualized using a fluorescence microscope.

\section{Computerized Morphometric Analysis}

The thickness of submesothelial compact zone between basal border of surface mesothelial cells and upper border of peritoneal adipose tissue was estimated by computerized morphometric analysis of Masson's trichrome-stained micrographs with $200 \times$ original magnification using the ImageJ analysis program, version 1.52a (National Institutes of Health, Bethesda, MD, USA). Five portions were randomly selected for the measurement and calculation of the average thickness of the submesothelial compact zone for each sample based on the five-point measurement method reported by Honda et al. (24).

\section{Statistical Analyses}

The integrated pixel density of immunoblot bands was determined using a densitometer and the ImageJ analysis program. All in vitro studies were repeated at least three times. All data are expressed as mean \pm SD. Differences in means were tested in Graphpad Prism 8 or SPSS 24. One-way ANOVA tests were performed for multiple comparisons of values followed by Tukey's post-hoc test. $P<0.05$ was considered to represent a statistically significant difference.

\section{RESULTS}

\section{Peritoneal Fibrosis in the Mouse Model of Peritoneal Dialysis Is Improved by Sal A Treatment, Concomitant With the Attenuation of GSK3 $\beta$ Hyperactivity}

Long-term stimulation by PDF has been associated with peritoneal fibrosis. Indeed, peritoneal fibrosis was evident in the mouse model of peritoneal dialysis on week 7 , as revealed by Masson trichrome staining for collagens (Figure 2A).
Computerized morphometric analysis demonstrated a marked thickening of the submesothelial compact zone as compared with PBS-treated mice (Figure 2B). Morphologic findings were corroborated by immunoblot analysis for peritoneal tissues, which demonstrated an increased expression of fibronectin, a key component of the extracellular matrix, vimentin, the intermediate filament and mesenchymal marker, and the profibrotic cytokine PAI-1 (Figures 2C,D). Sal A treatment significantly diminished collagen deposition in peritoneal tissues as shown by Masson's Trichrome staining, lessened the thickness of the submesothelial compact zone, and mitigated the PDF induced overexpression of fibronectin, vimentin and PAI1 in peritoneal tissues as shown by immunoblot analysis (Figure 2). GSK3 $\beta$ has been implicated in tissue fibrogenesis in diverse organ systems. In consistency, the above findings of PDF-elicited peritoneal fibrosis were associated with GSK3 $\beta$ hyperactivity, marked by increased GSK3 $\beta$ expression and reduced inhibitory phosphorylation of GSK3 $\beta$ at serine 9 residue, as revealed by immunoblot analysis of peritoneal tissues (Figures 2C,D). In contrast, the PDF-triggered GSK3 $\beta$ hyperactivity was averted after Sal A, concomitant with the protective effect on peritoneal fibrosis.

\section{Sal A Treatment Reinforces the Nrf2 Antioxidant Response and Diminishes Peritoneal Oxidative Injury}

PDF is known to be able to instigate oxidative stress via various mechanisms such as hypertonicity. To ascertain if this resulted in any oxidative injury in the peritoneal tissues, fluorescent immunohistochemistry staining was performed. Shown in Figures 3A,B, PDF treatment caused a drastic induction of nitrotyrosine, a reliable in vivo marker of oxidative stress associated protein modification and dysfunction. Sal A treatment significantly reduced the expression of nitrotyrosine in PDF-stimulated peritoneum, denoting an antioxidant and protective activity. The histologic findings were consistently corroborated by immunoblot analysis of peritoneal tissues for nitrotyrosine combined with densitometry (Figure 3C). Upon oxidative stress, Nrf2 signaling will be immediately ignited 


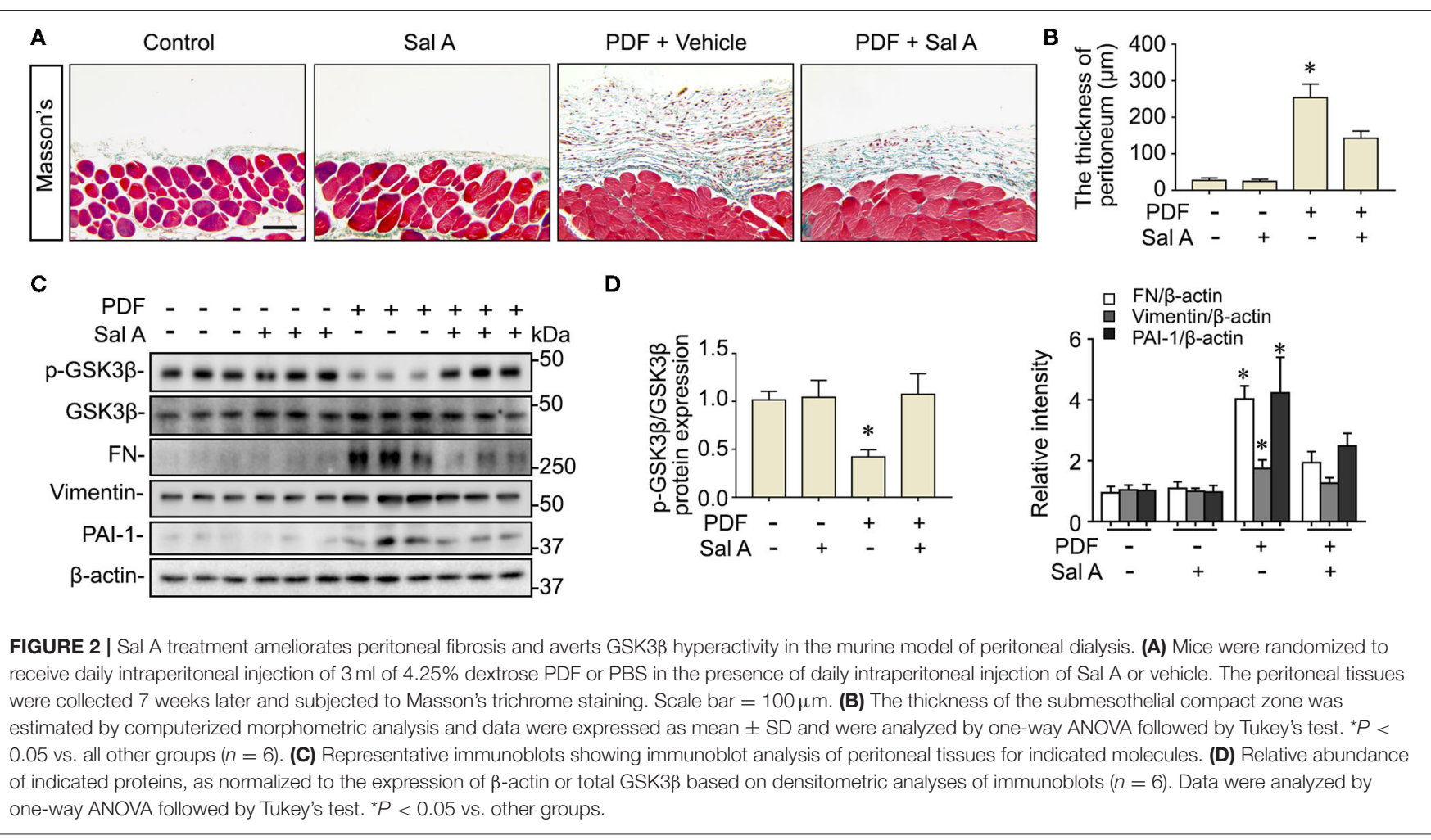

as the master regulator of anti-oxidative responses and the primary cellular defense signaling against the cytotoxic effects of reactive oxygen species (ROS) and reactive nitrogen oxide species (RNOS). To determine if the Sal A conferred beneficial action on peritoneal oxidative injury stems from a possible effect on the Nrf2 antioxidant response, peritoneal tissues were subjected to fluorescent immunohistochemistry for Nrf2 (Figure 3A). Under basal conditions, Nrf2 staining was weak and mostly located to cytoplasm in peritoneal tissues. Upon PDF stimulation, there was a remarkable induction of $\mathrm{Nrf} 2$ response as a self-defense mechanism, shown by increased Nrf2 expression with some nuclear staining denoting nuclear translocation. Sal A treatment greatly enhanced Nrf2 antioxidant response, marked by reinforced Nrf2 nuclear translocation (Figures 3A,B). To quantify the histologic findings, nuclear fractions of peritoneal tissues were prepared and subjected to immunoblot analysis in combination with densitometry, which consistently demonstrated that nuclear expression of $\mathrm{Nrf} 2$ in PDF-treated peritoneal tissues was significantly potentiated by Sal A (Figures 3C,D).

\section{NFkB Activation in Peritoneal Tissues of Mouse Models of Peritoneal Dialysis Is Mitigated by Sal A, Resulting in Improved Peritoneal Inflammation, and Maladaptive Angiogenesis}

In addition to oxidative injury, PDF is also known to elicit peritoneal inflammation, which may be both the cause and consequence of peritoneal fibrosis. Indeed, fluorescent immunohistochemistry for CD45, the leukocyte common antigen, and CD68, a specific marker for monocytes/macrophages, revealed prominent inflammatory infiltration in peritoneal tissues stimulated by PDF (Figure 4A). This effect was markedly suppressed by Sal A treatment. The morphologic findings were further confirmed by absolute counting of CD45 and CD68 positive cells (Figure 4B). In consistency, the proinflammatory cytokines induced by PDF stimulation like TNF- $\alpha$, IL-1 $\beta$, and MCP- 1 were significantly suppressed by Sal A treatment, as measured by ELISA (Figure 4C). Central to the pathogenic mechanisms of inflammation, NFкB pathway plays a key role. As shown by immunoblot analysis of peritoneal tissues followed by densitometry (Figures 4D,E), activation of NFKB, marked by phosphorylation of NFKB RelA/p65, was triggered by PDF stimulation, and this effect was substantially abrogated after Sal A treatment. As a pathological event closely associated with inflammation and NFкB activation, maladaptive angiogenesis also plays an instrumental role in peritoneal fibrosis. As shown by fluorescent immunohistochemistry for CD31, a standard marker for endothelial cells, PDF injury resulted in evident increase of the amount of endothelial cells in peritoneal tissues, denoting maladaptive angiogenesis (Figure 5A). This effect was considerably suppressed by Sal A treatment. Moreover, the VEGF positive cells were significantly increased in mice treated with PDF based on immunohistochemistry staining, while Sal A markedly reduced the amount of VEGF positive cells. The morphologic findings were further verified by calculating 


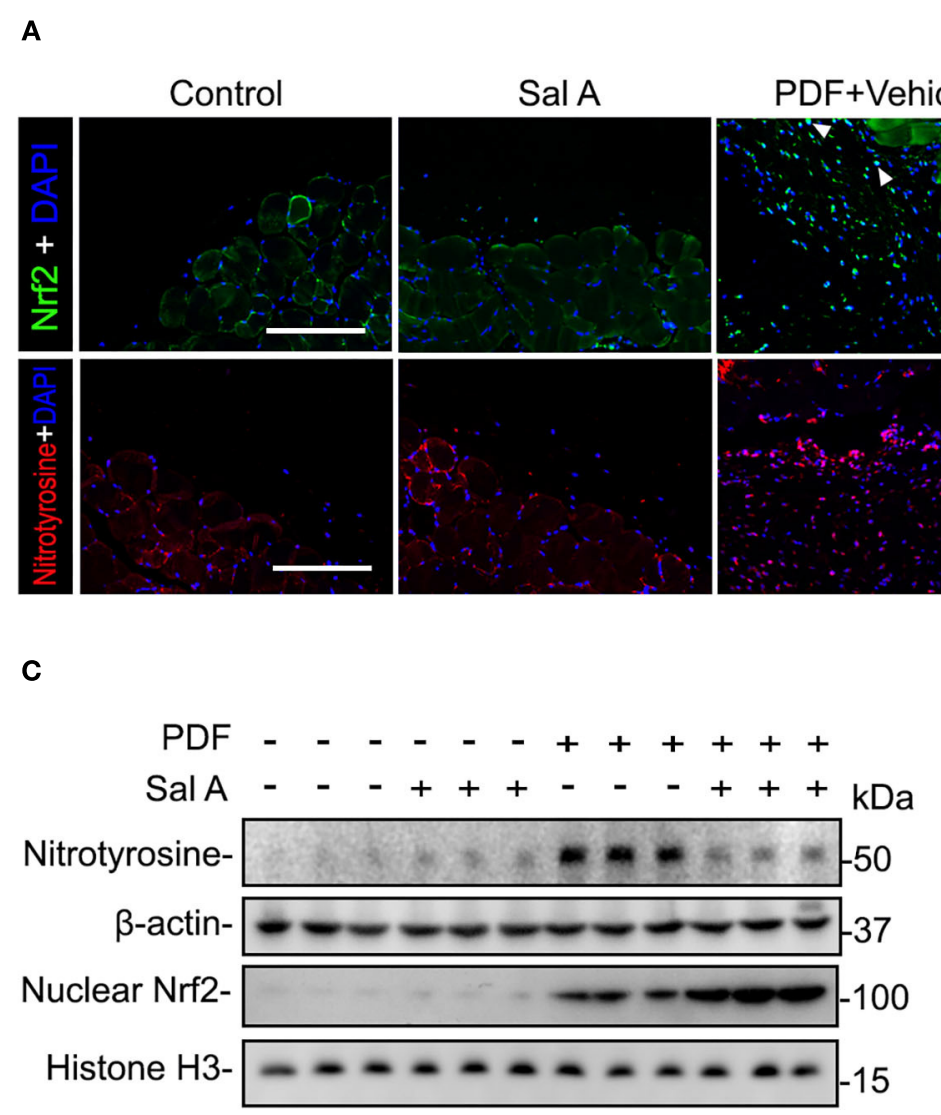

B

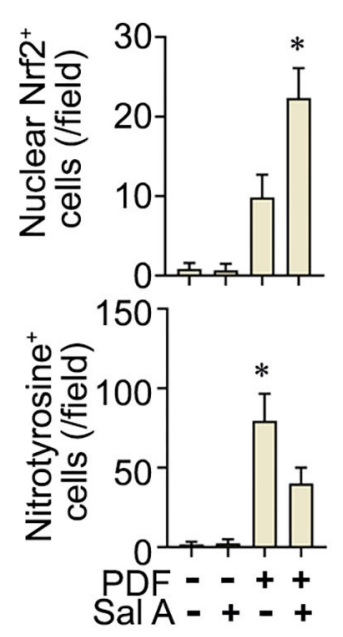

D

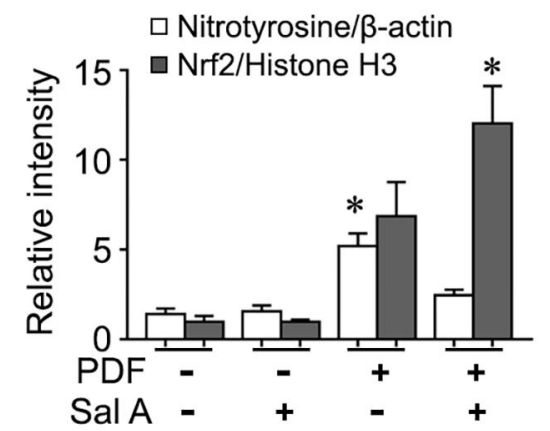

FIGURE 3 | Sal A treatment of murine models of peritoneal dialysis reinforces Nrf2 antioxidant response and diminishes oxidative damage in peritoneum. (A) Mice were treated as elaborated in Figure 2. The peritoneal tissues were collected on week 7 and subjected to fluorescence immunohistochemistry staining for indicated proteins. The arrowheads indicate nuclear Nrf2 positive cells. Scale bar $=200 \mu \mathrm{m}$. (B) Absolute counting of nuclear Nrf2 or nitrotyrosine positive (+) cells. Data were analyzed by one-way ANOVA followed by Tukey's test. ${ }^{*}<0.05$ vs. other groups $(n=6)$. (C) Representative immunoblots showing immunoblot analysis of peritoneal tissues for indicated molecules. (D) Relative abundance of indicated proteins, as normalized to that of $\beta$-actin or histone based on densitometric analyses of immunoblots $(n=6)$. Data were analyzed by one-way ANOVA followed by Tukey's test. ${ }^{\star} P<0.05$ vs. other groups.

the CD31 positive area per microscopic field and by absolute counting of VEGF positive cells (Figure 5B).

\section{GSK3 $\beta$ Inhibition Mediates the Effect of Sal A on Nrf2 Response and NFKB Signaling in Peritoneal Mesothelial Cells Upon PDF Injury}

Evidence suggests that GSK3 $\beta$ is a convergent point of the signaling cascades regulating both $\mathrm{Nrf2}$ and $\mathrm{NF \kappa} B$ pathways and thus is likely involved in antioxidant response and inflammation. To discern if there is a causal relationship between the above-observed inhibitory effect of Sal A treatment on PDFelicited GSK3 $\beta$ hyperactivity and the beneficial effect of Sal A on oxidative injury and inflammation, primary cultures of PMC were employed and treated with medium containing hypertonic (4.25\%) dextrose to model PDF-elicited peritoneal fibrosis in vitro (Figure 6). Reminiscent of the key features of PDF-elicited peritoneal fibrosis, hypertonic dextrose elicited considerable profibrotic changes, inflammatory reaction and oxidative damage in control empty vector-transfected PMC cultures, marked, respectively, by fibronectin overexpression, increased phosphorylation and activation of NFאB RelA/p65, and induction of nitrotyrosine, concomitant with an antioxidant selfdefense as evidenced by increased nuclear expression of Nrf2. In consistency, the injurious effects of hypertonic dextrose were associated with GSK3 $\beta$ hyperactivity, marked by elevated GSK3 $\beta$ expression and reduced inhibitory phosphorylation of GSK3 $\beta$ at serine 9 residue. In contrast, Sal A treatment averted the GSK3 $\beta$ hyperactivity triggered by hypertonic dextrose. Accordingly, these injurious effects of hypertonic dextrose were substantially abolished by Sal A treatment, in parallel with an enhanced nuclear expression of Nrf2, indicative of a reinforced antioxidant response (Figure 6). In contrast, in PMC cultures expressing the constitutively active GSK3 $\beta$ mutant as shown by immunoblot analysis for HA, the protective effect of Sal A against the hypertonic dextrose instigated profibrotic changes, inflammatory reaction, and oxidative damage was largely blunted (Figure 6). These findings suggest that Sal A potentiates Nrf2 response and mitigates NFKB signaling via GSK3 $\beta$ inhibition. 

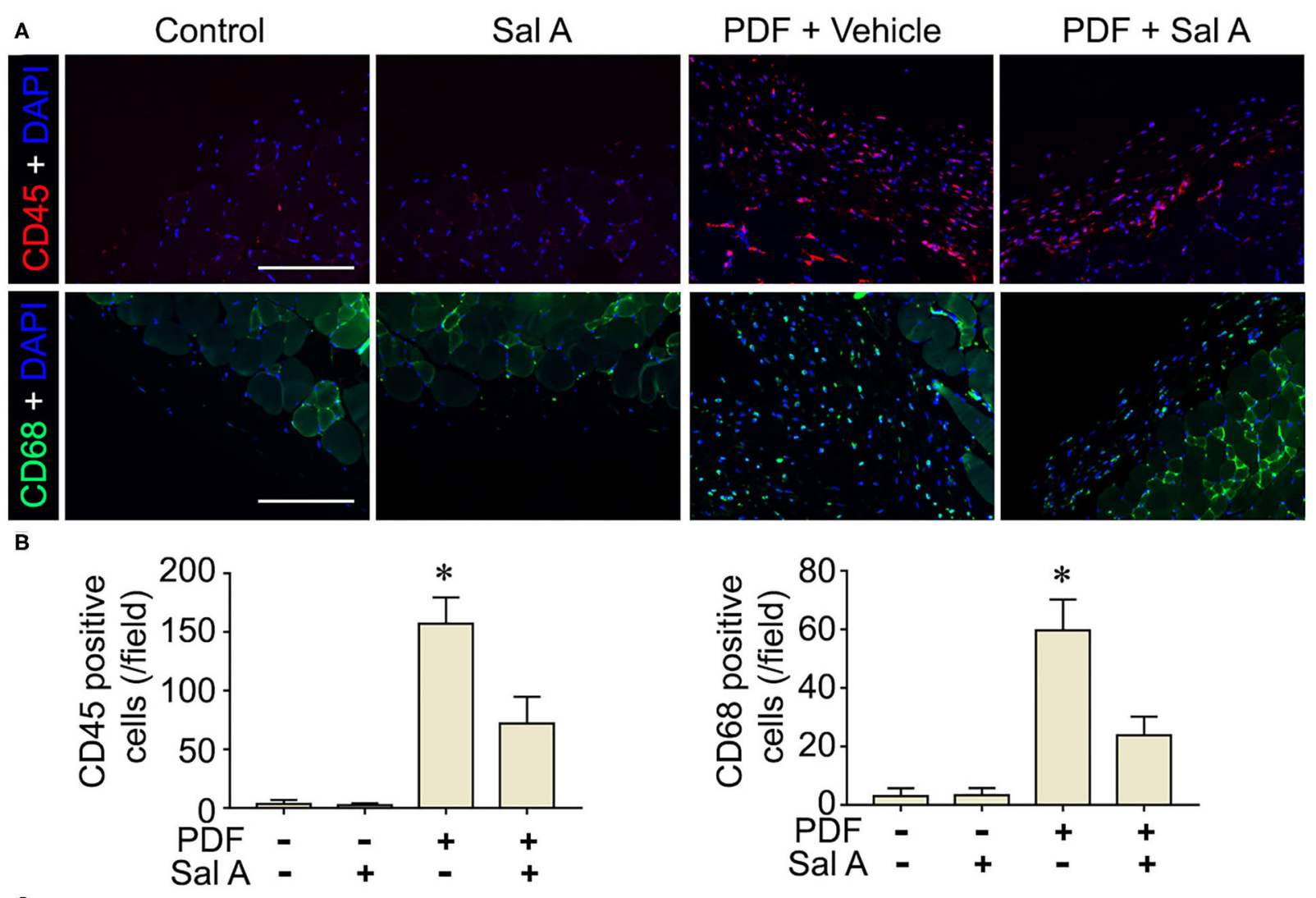

C
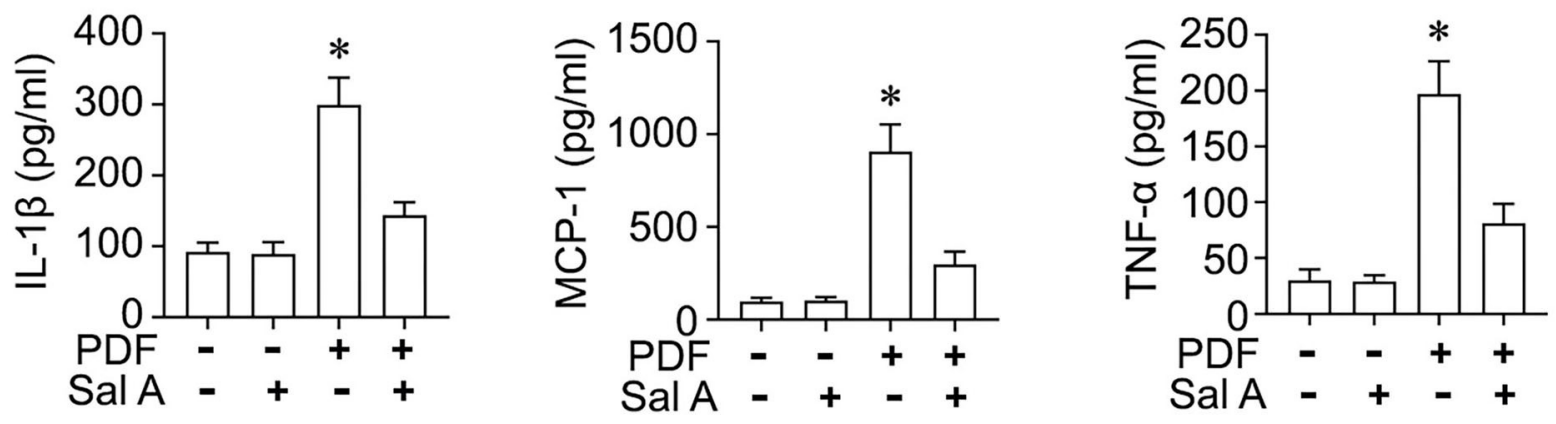

D

E
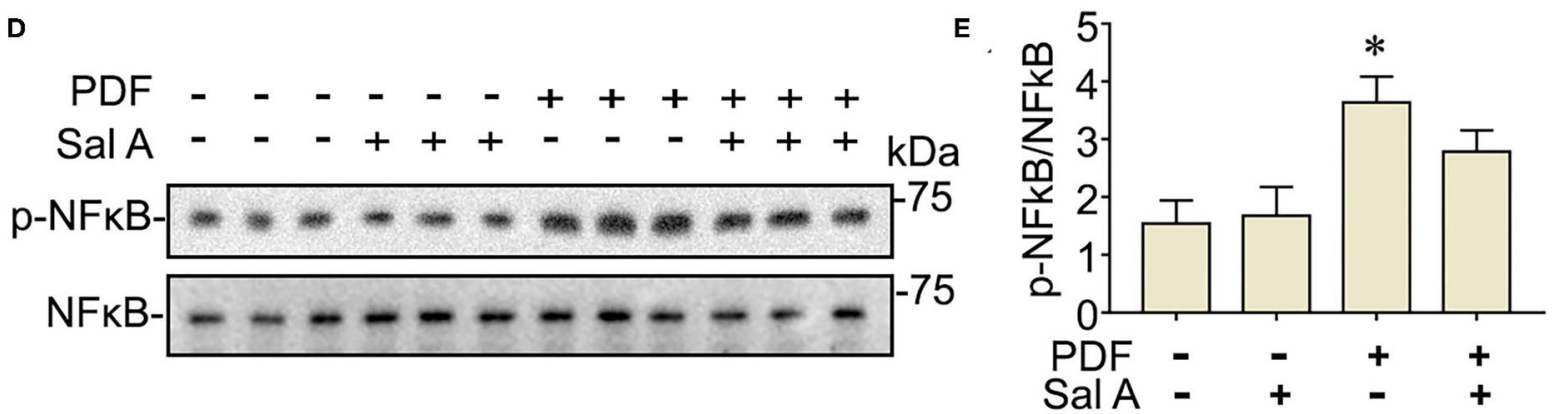

FIGURE 4 | Sal A treatment of murine models of peritoneal dialysis attenuates inflammation and NFKB activation in peritoneum. (A) Mice were treated as elaborated in Figure 2. The peritoneal tissues were collected on week 7 and subjected to immunofluoresence staining for CD45 or CD68 with nuclear counterstaining of DAPI. Scale bar $=200 \mu \mathrm{m}$. (B) Absolute counting of CD45 or CD68 positive cells. Data were analyzed by one-way ANOVA followed by Tukey's test. ${ }^{*} P<0.05$ vs. other groups $(n$ $=6$ ). (C) The expression levels of IL-1 $\beta$, MCP-1, and TNF- $\alpha(n=4)$. Data were analyzed by one-way ANOVA followed by Tukey's test. * $P<0.05$ vs. other groups. (D) Representative immunoblots showing immunoblot analysis of peritoneal tissues for indicated molecules. (E) Relative abundance of p-NFkB p65, as normalized to that of NFkB p65 based on densitometric analyses of immunoblots $(n=6)$. Data were analyzed by one-way ANOVA followed by Tukey's test. ${ }^{*} P<0.05$ vs. other groups. 


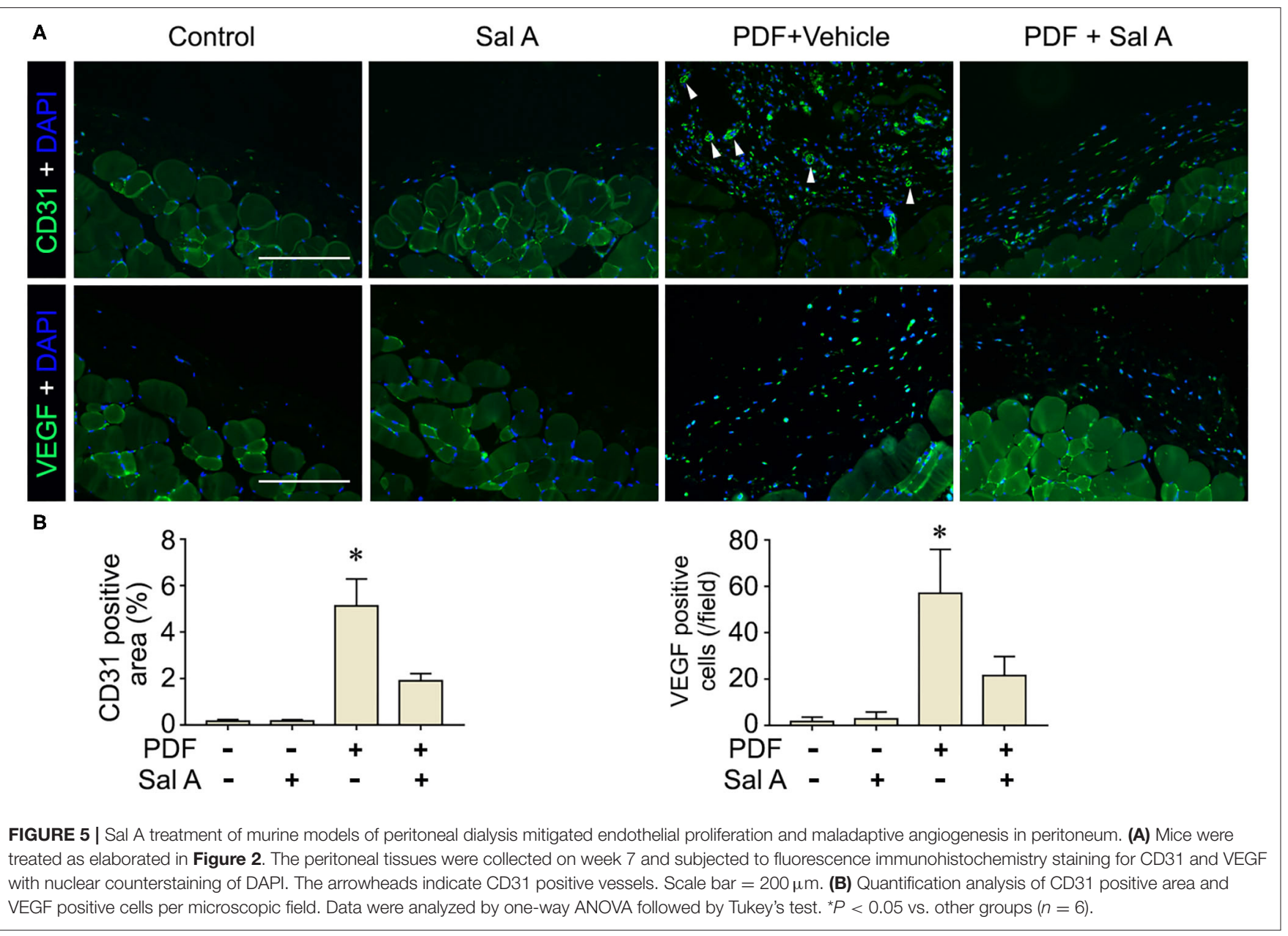

\section{DISCUSSION}

Peritoneal fibrosis is a common and devastating complication in patients undergoing peritoneal dialysis, and a major cause responsible for peritoneal membrane ultrafiltration dysfunction and failure of peritoneal dialysis (1). Our present study demonstrated that Sal A, an active component of $S$. miltiorrhiza, ameliorates peritoneal fibrosis by mitigating oxidative injury and inflammation in a mouse model of peritoneal dialysis. Accumulating evidence recently suggests that S. miltiorrhiza exerts a unique anti-fibrotic effects in pre-clinical models of tissue fibrogenesis in a number of organ systems, including the heart (25), lung (26), liver (27), and kidney (28). However, its role in peritoneal fibrosis is unknown. To the best of knowledge, this is the first study demonstrating that the active component of S. miltiorrhiza Sal A possesses a potent protective effect against peritoneal fibrosis in vivo in mouse models of peritoneal dialysis and in vitro in peritoneal mesothelial cells stimulated by hypertonic dextrose.

Regardless of the original etiology, diseases in different organ systems progress via the final common pathways that include fibrogenesis, inflammation and oxidative injury (3).
The beneficial effect of $S$. miltiorrhiza on multiple experimental disease models has been associated with improvement in these final common pathways (25-28). Nevertheless, the underlying molecular mechanisms are controversial. There is evidence suggesting that $S$. miltiorrhiza is able to intercept a number of cell signaling cascades that modify these pathologic pathways. For instance, in rat PMC exposed to high ambient glucose, S. miltiorrhiza injection was able to attenuate the loss of E-cadherin mRNA expression and repress the induction of mRNA expression of a-smooth muscle actin, suggesting that S. miltiorrhiza has an anti-fibrotic effect in peritoneal fibrosis (29). In addition, in murine models of Gynura segetum-induced hepatic sinusoidal obstruction syndrome, S. miltiorrhiza was shown to confer a hepatoprotective effect that mitigated the expression of proinflammatory mediators, like TNF-a, VCAM1 , and ICAM-1 in diseased livers (30). This protective effect of $S$. miltiorrhiza was found to be associated with reduced activity of NFKB p65 (30). This is in line with the observation by Yang et al. (31), in which administration of S. miltiorrhiza extract reduced myocardial infarction-induced inflammation, as evidenced by the decreased expression of proinflammatory cytokines such as IL-1 $\beta$, TNF, and IL- 6 . In consistency, the present study also demonstrated an inhibitory effect of Sal 

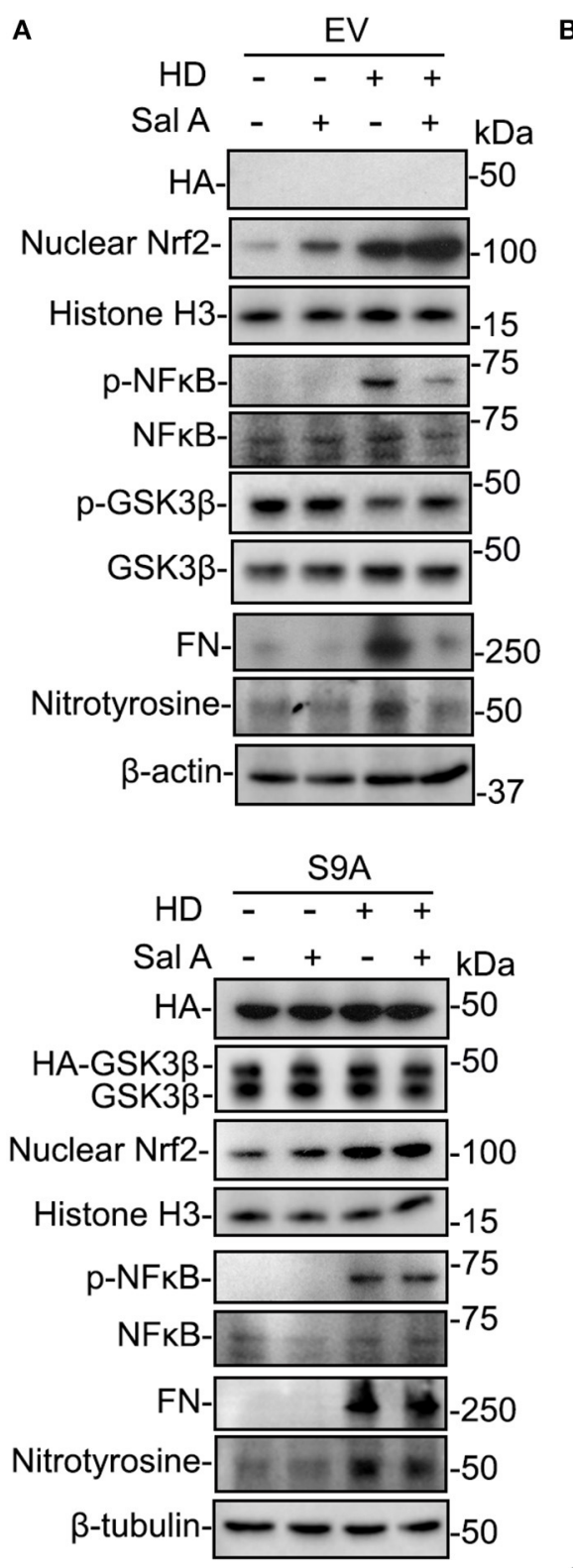
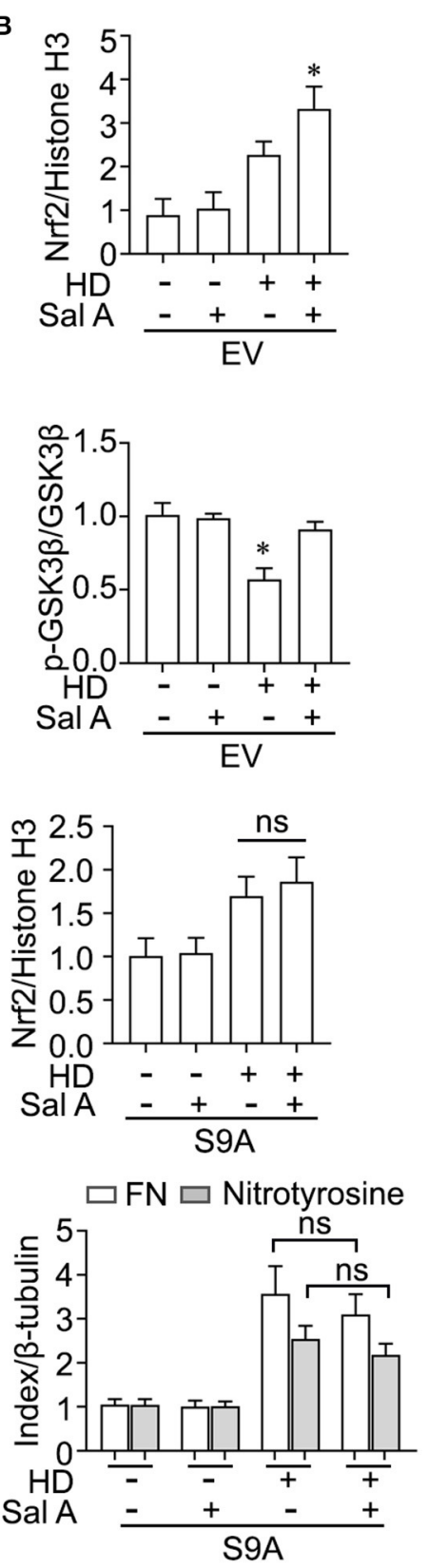
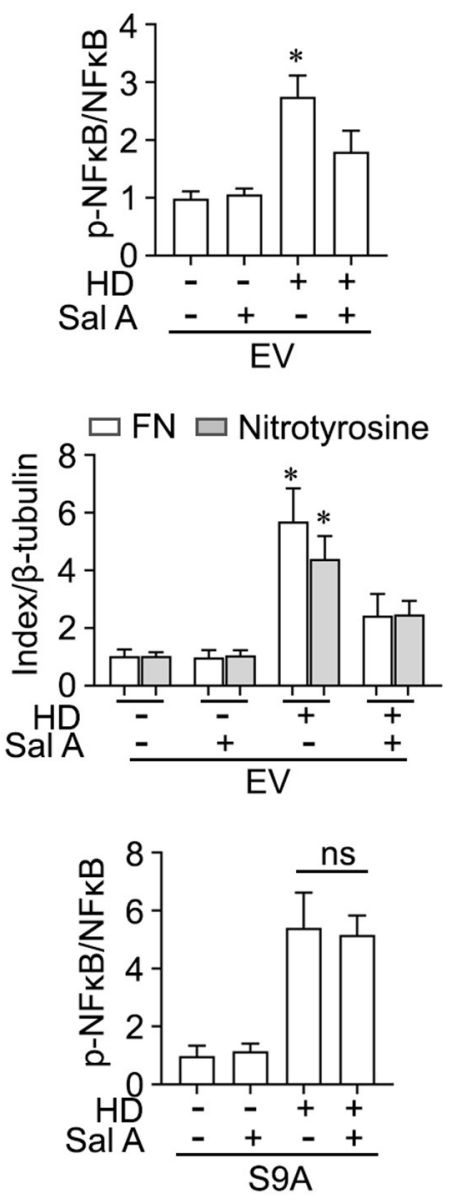

FIGURE 6 | GSK3 $\beta$ inhibition is required for the regulatory effect of Sal A on NFkB and Nrf2 activation in cultured peritoneal mesothelial cells exposed to hypertonic dextrose. (A) Primary cultures of murine peritoneal mesothelial cells were transfected with the empty vector (EV) or the vector encoding the HA-conjugated constitutively active mutant of GSK3 $\beta$ (S9A) and then were exposed to hypertonic dextrose (HD) or vehicle treatment for $48 \mathrm{~h}$. Representative immunoblots showing immunoblot analysis of collected cells for indicated molecules. (B) Relative abundance of indicated proteins, as normalized to the expression of histone $\mathrm{H} 3$, NFKB, total GSK3 $\beta, \beta$-actin or $\beta$-tubulin based on densitometric analyses of immunoblots $(n=3)$. Data were analyzed by one-way ANOVA followed by Tukey's test. ${ }^{\star} P<$ 0.05 vs. other groups. ns, not significant.

A on $\mathrm{NF}_{\kappa} \mathrm{B}$ activation in peritoneum of peritoneal dialysis models, associated with amelioration of inflammatory infiltration and maladaptive endothelial proliferation and angiogenesis. Apart from the anti-inflammatory property, S. miltiorrhiza has been noted to have a potent antioxidant activity. To this end, in rats with paracetamol-induced liver injury, $S$. miltiorrhiza was shown to significantly heighten the ratios of reduced glutathione (GSH) to oxidized glutathione (GSSG), denoting an antioxidant effect (32). In agreement, Wang et al. (33) found that treatment with extracts of S. miltiorrhiza increased the expression of heme-oxygenase-1, $\mathrm{NAD}(\mathrm{P}) \mathrm{H}$ quinine oxidoreductase, and Nrf2 in the injured brain tissues 


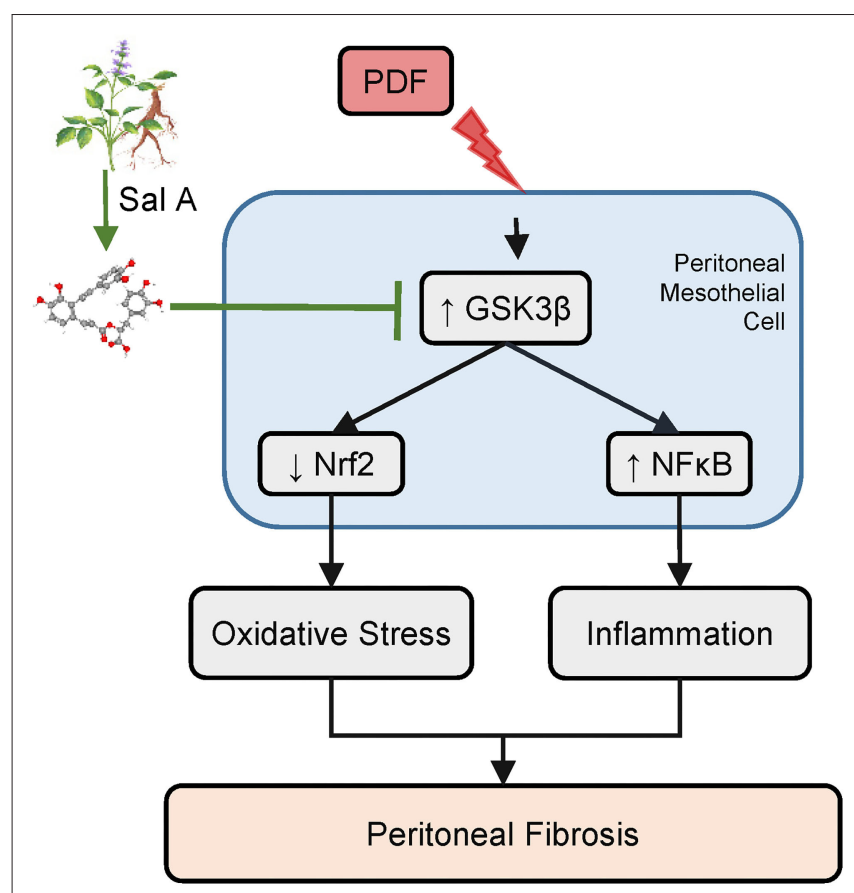

FIGURE 7 | A schematic diagram depicts the molecular mechanism underlying the protective effect of Sal A, the active ingredient of Salvia miltiorrhiza, on peritoneal fibrosis, inflammation, and oxidative injury in murine models of peritoneal dialysis. As a key active component of Salvia miltiorrhiza, Sal $A$ is able to directly bind to and inhibit GSK3 $\beta$ (14). GSK3 $\beta$ is a convergent point of myriad pathways modifying the severity of peritoneal fibrosis, including the NFKB directed inflammatory response pathway and the Nrf2 antioxidant pathway. Inhibition of GSK3 $\beta$ by Sal A mitigates NFKB phosphorylation and activation and reinforces Nrf2 antioxidant self-defense in peritoneal mesothelial cells and thereby attenuates inflammation, maladaptive angiogenesis and oxidative damage in peritoneum exposed to the hypertonic PDF, resulting in an improved peritoneal fibrosis.

and resulted in a neuroprotective effect against cerebral ischemia injury in rats. Our findings here consistently suggest an antioxidant activity of $S$. miltiorrhiza, as evidenced by the reinforced Nrf2 antioxidant response and diminished expression of nitrotyrosine.

In light of the multipronged effect of S. miltiorrhiza on various pathologic pathways like inflammation and oxidative stress, it is tempting to speculate that the active ingredient of $S$. miltiorrhiza that mediates the therapeutic action may possibly target some regulatory signaling transducers upstream of and common to diverse pathologic pathways. Among these regulatory signaling transducers, GSK3 $\beta$ has emerged as a crucial modifier of a myriad of disease processes, including inflammation, oxidative stress, fibrogenesis and tissue injury, repair and injury $(21,34,35)$. GSK3 $\beta$ was originally identified to be a critical mediator of the insulin and glycogen biosynthesis pathway (34). Later, more and more data revealed that it is also a key kinase regulating the activity of many other signaling pathways (34), including the $\mathrm{Wnt} / \beta$-catenin pathway, the Hedgehog signaling, the Notch pathway and cytoskeleton integrity. More recently, GSK3 $\beta$ has been implicated in the regulation of proinflammatory $\mathrm{NF \kappa B}$ activity (36). It was found that GSK3 $\beta$ was sufficient and essential for NFKB RelA/p65 phosphorylation, which specifies the expression of selective $\mathrm{NF} \kappa \mathrm{B}$ target molecules, including specifically proinflammatory cytokines and mediators (37). As such, GSK3 $\beta$-dicated finetuning of NFкB plays a key role in controlling the severity of inflammatory injury (7). In addition, Nrf2 is a cognate substrate for GSK3 $\beta$ (38). GSK3 $\beta$-directed nuclear exclusion and degradation of Nrf2 is pivotal in switching off the selfprotective antioxidant stress response after injury (39). Thus, GSK3 $\beta$-directed regulation of Nrf2 represents a key mechanism of control of the magnitude and duration of the stress elicited Nrf2 antioxidant response at a delayed/late phase following insults (40). Several lines of evidence from our study suggests that $S$. miltiorrhiza may target GSK3 $\beta$ to exert its beneficial action in diseases. First, the active component of $S$. miltiorrhiza Sal A was able to avert GSK3 $\beta$ hyperactivity in peritoneal tissues exposed to PDF, concomitant with the anti-fibrotic, antiinflammatory and antioxidant effect. In addition, the protective effect of Sal A on peritoneal fibrosis was recapitulated in vitro in peritoneal mesothelial cells injured with hypertonic dextrose and abolished by overexpression of the constitutively active GSK3 $\beta$. Therefore, we posited that Sal A is likely to have a GSK3 $\beta$ inhibitory activity (Figure 7). In support of our findings, Zhang et al. (13) demonstrated that Sal A was able to protect against progressive chronic kidney injury in in 5/6 nephrectomized rats by activating the GSK3 $\beta / \mathrm{Nrf} 2$ signaling pathway and inhibiting the NFKB. It is not clear if Sal A directly inhibits GSK3 $\beta$ activity or indirectly via other mediators. However, there is evidence favoring the former postulation. By using the automated high-throughput screening Kinase-Glo luminescent kinase assay platform, Paudel et al. (14) found that Sal A is an ATPcompetitive inhibitor of GSK3 $\beta$ with an inhibitory potency comparable to standard small molecule inhibitors of GSK3 $\beta$. The mechanism of action was revealed by in silico modeling to be the hydrophobic, $\pi$-cation, and hydrophilic interactions of Sal A at ATP and substrate sites of GSK3 $\beta$. Admittedly, the present study is a pilot one with a short period in animal models. Further studies are merited to ascertain the safety and efficacy of Sal A therapy in preventing peritoneal fibrosis in patients undergoing peritoneal dialysis. Admittedly, S. miltiorrhiza also contains other active components, such as Tanshinone, which has been shown to be protective against peritoneal fibrosis (41). In addition, multiple other components of $S$. miltiorrhiza also have been shown to prevent peritoneal adhesions (42). In light of the molecular mechanism uncovered by the present study, it merits further studies to determine if GSK3 $\beta$ signaling pathway is involved in the beneficial activities of other components of S. miltiorrhiza.

In summary, Sal A, the active ingredient of S. miltiorrhiza ameliorates peritoneal fibrosis and mitigated oxidative injury and inflammation in vivo in a mouse model of peritoneal dialysis and in vitro in peritoneal mesothelial cells exposed to hypertonic dextrose. The beneficial action of Sal A is 
likely mediate via at least in part GSK3 $\beta$ inhibition. Our findings may pave the way for developing a novel therapy for preventing peritoneal fibrosis and ultrafiltration failure in peritoneal dialysis.

\section{DATA AVAILABILITY STATEMENT}

The raw data supporting the conclusions of this article will be made available by the authors, without undue reservation.

\section{ETHICS STATEMENT}

The animal study was reviewed and approved by the Ethics Committee of the First Affiliated Hospital of Zhengzhou University.

\section{REFERENCES}

1. Zhou Q, Bajo MA, Del Peso G, Yu X, Selgas R. Preventing peritoneal membrane fibrosis in peritoneal dialysis patients. Kidney Int. (2016) 90:51524. doi: 10.1016/j.kint.2016.03.040

2. Raglianti V, Rossi GM, Vaglio A. Idiopathic retroperitoneal fibrosis: an update for nephrologists. Nephrol Dial Transplant. (2020) 36:177381. doi: 10.1093/ndt/gfaa083

3. Wynn TA, Ramalingam TR. Mechanisms of fibrosis: therapeutic translation for fibrotic disease. Nat Med. (2012) 18:1028-40. doi: 10.1038/nm.2807

4. Ahmed SM, Luo L, Namani A, Wang XJ, Tang X. Nrf2 signaling pathway: pivotal roles in inflammation. Biochim Biophys Acta Mol Basis Dis. (2017) 1863:585-97. doi: 10.1016/j.bbadis.2016.11.005

5. Margetts PJ, Kolb M, Yu L, Hoff CM, Holmes CJ, Anthony DC, et al. Inflammatory cytokines, angiogenesis, and fibrosis in the rat peritoneum. Am J Pathol. (2002) 160:2285-94. doi: 10.1016/S0002-9440(10)61176-5

6. Wardyn JD, Ponsford AH, Sanderson CM. Dissecting molecular cross-talk between Nrf2 and NF-kappaB response pathways. Biochem Soc Trans. (2015) 43:621-6. doi: 10.1042/BST20150014

7. Liu Z, Gong R. Remote ischemic preconditioning for kidney protection: GSK3 $\beta$-centric insights into the mechanism of action. Am J Kidney Dis. (2015) 66:846-56. doi: 10.1053/j.ajkd.2015.06.026

8. Lu M, Wang P, Qiao Y, Jiang C, Ge Y, Flickinger B, et al. GSK3 $\beta$-mediated Keap1-independent regulation of Nrf2 antioxidant response: a molecular rheostat of acute kidney injury to chronic kidney disease transition. Redox Biol. (2019) 26:101275. doi: 10.1016/j.redox.2019.101275

9. Zheng H, Yang Z, Xin Z, Yang Y, Yu Y, Cui J, et al. Glycogen synthase kinase3 $\beta$ : a promising candidate in the fight against fibrosis. Theranostics. (2020) 10:11737-53. doi: 10.7150/thno.47717

10. Rusai K, Herzog R, Kuster L, Kratochwill K, Aufricht C. GSK-3 $\beta$ inhibition protects mesothelial cells during experimental peritoneal dialysis through upregulation of the heat shock response. Cell Stress Chaperones. (2013) 18:569-79. doi: 10.1007/s12192-013-0410-6

11. Su CY, Ming QL, Rahman K, Han T, Qin LP. Salvia miltiorrhiza: traditional medicinal uses, chemistry, and pharmacology. Chin J Nat Med. (2015) 13:16382. doi: 10.1016/S1875-5364(15)30002-9

12. Wang L, Ma R, Liu C, Liu H, Zhu R, Guo S, et al. Salvia miltiorrhiza: a potential red light to the development of cardiovascular diseases. Curr Pharm Des. (2017) 23:1077-97. doi: 10.2174/138161282266616101010 5242

13. Zhang HF, Wang JH, Wang YL, Gao C, Gu YT, Huang J, et al. Salvianolic acid A protects the kidney against oxidative stress by activating the Akt/GSK-3 $\beta / \mathrm{Nrf2}$ signaling pathway and inhibiting the NF-kappaB signaling pathway in 5/6 nephrectomized rats. Oxid Med Cell Longev. (2019) 2019:2853534. doi: 10.1155/2019/2853534

\section{AUTHOR CONTRIBUTIONS}

PW and FZ contributed to the study design. FZ, LY, and XL carried out animal and cell culture experiments. FZ, YL, and $\mathrm{XH}$ performed the data analysis. FZ and PW contributed to the manuscript editing. All authors have read and agreed to the published version of the manuscript.

\section{FUNDING}

This work was supported in part by the National Natural Science Foundation of China Grants 81873612 and 81770672 (to PW) and Foundation of He'nan Educational Committee Grants 20B320040 and 19B320019 (to XH). The funders had no role in the design and conduct of this study, collection and interpretation of the data, or preparation and approval of the manuscript.

14. Paudel P, Seong SH, Zhou Y, Park CH, Yokozawa T, Jung HA, et al. Rosmarinic acid derivatives' inhibition of glycogen synthase kinase- $3 \beta$ is the pharmacological basis of Kangen-Karyu in Alzheimer's Disease. Molecules. (2018) 23:2919. doi: 10.3390/molecules23112919

15. Ma L, Tang L, Yi Q. Salvianolic acids: potential source of natural drugs for the treatment of fibrosis disease and cancer. Front Pharmacol. (2019) 10:97. doi: 10.3389/fphar.2019.00097

16. Liu P, Hu Y, Liu C, Liu C, Zhu D. Effects of salviainolic acid A (SA-A) on liver injury: SA-A action on hepatic peroxidation. Liver. (2001) 21:38490. doi: 10.1034/j.1600-0676.2001.210604.x

17. Pan Y, Fu H, Kong Q, Xiao Y, Shou Q, Chen H, et al. Prevention of pulmonary fibrosis with salvianolic acid a by inducing fibroblast cell cycle arrest and promoting apoptosis. J Ethnopharmacol. (2014) 155:158996. doi: 10.1016/j.jep.2014.07.049

18. Yu J-W, Duan W-J, Huang X-R, Meng X-M, Yu X-Q, Lan H-Y. MicroRNA$29 \mathrm{~b}$ inhibits peritoneal fibrosis in a mouse model of peritoneal dialysis. Lab Invest. (2014) 94:978-90. doi: 10.1038/labinvest.2014.91

19. Zeng X, Chen X, Qin H, Han Y, Chen X, Han Z, et al. Preventive effects of a natural anti-inflammatory agent salvianolic acid A on acute kidney injury in mice. Food Chem Toxicol. (2020) 135:110901. doi: 10.1016/j.fct.2019.110901

20. Bot J, Whitaker D, Vivian J, Lake R, Yao V, McCauley R. Culturing mouse peritoneal mesothelial cells. Pathol Res Pract. (2003) 199:3414. doi: 10.1078/0344-0338-00427

21. $\mathrm{Xu} \mathrm{W,} \mathrm{Ge} \mathrm{Y,} \mathrm{Liu} \mathrm{Z,} \mathrm{Gong} \mathrm{R.} \mathrm{Glycogen} \mathrm{synthase} \mathrm{kinase} 3 \beta$ dictates podocyte motility and focal adhesion turnover by modulating paxillin activity: implications for the protective effect of low-dose lithium in podocytopathy. Am J Pathol. (2014) 184:2742-56. doi: 10.1016/j.ajpath.2014.06.027

22. Yang LL, Li DY, Zhang YB, Zhu MY, Chen D, Xu TD. Salvianolic acid A inhibits angiotensin II-induced proliferation of human umbilical vein endothelial cells by attenuating the production of ROS. Acta Pharmacol Sin. (2012) 33:41-8. doi: 10.1038/aps.2011.133

23. $\mathrm{Xu} \mathrm{W,} \mathrm{Ge} \mathrm{Y,} \mathrm{Liu} \mathrm{Z,} \mathrm{Gong} \mathrm{R.} \mathrm{Glycogen} \mathrm{synthase} \mathrm{kinase} 3 \beta$ orchestrates microtubule remodeling in compensatory glomerular adaptation to podocyte depletion. J Biol Chem. (2015) 290:1348-63. doi: 10.1074/jbc.M114.5 93830

24. Honda K, Hamada C, Nakayama M, Miyazaki M, Sherif AM, Harada T, et al. Impact of uremia, diabetes, and peritoneal dialysis itself on the pathogenesis of peritoneal sclerosis: a quantitative study of peritoneal membrane morphology. Clin J Am Soc Nephrol. (2008) 3:720-8. doi: 10.2215/CJN.03630807

25. Zhang Y, Wang H, Cui L, Zhang Y, Liu Y, Chu X, et al. Continuing treatment with Salvia miltiorrhiza injection attenuates myocardial fibrosis in chronic iron-overloaded mice. PLoS ONE. (2015) 10:e0124061. doi: 10.1371/journal.pone.0124061

26. Peng LY, An L, Sun NY, Ma Y, Zhang XW, Liu WH, et al. Salvia miltiorrhiza restrains reactive oxygen species-associated pulmonary fibrosis 
via targeting Nrf2-Nox4 redox balance. Am J Chin Med. (2019) 47:111331. doi: 10.1142/S0192415X19500575

27. Nan JX, Park EJ, Kang HC, Park PH, Kim JY, Sohn DH. Anti-fibrotic effects of a hot-water extract from Salvia miltiorrhiza roots on liver fibrosis induced by biliary obstruction in rats. J Pharm Pharmacol. (2001) 53:197204. doi: 10.1211/0022357011775406

28. Li L, Zhang Y, Ma J, Dong W, Song Q, Zhang J, et al. Salvia miltiorrhiza injection ameliorates renal damage induced by lead exposure in mice. ScientificWorldJournal. (2014) 2014:572697. doi: 10.1155/2014/ 572697

29. Akita K, Yasaka K, Shirai T, Ishii T, Harigae H, Fujii H. Interferon $\alpha$ enhances B cell activation associated with FOXM1 induction: potential novel therapeutic strategy for targeting the plasmablasts of systemic lupus erythematosus. Front Immunol. (2020) 11:498703. doi: 10.3389/fimmu.2020. 498703

30. Yang L, Huo JR, Zhu HY, Chen Z, Wang XY. The effect of Salvia miltiorrhiza in a mouse model of hepatic sinusoidal obstruction syndrome induced by Gynura segetum. Rev Esp Enferm Dig. (2019) 111:8237. doi: 10.17235/reed.2019.6085/2018

31. Yang J, Wang B, Li N, Zhou Q, Zhou W, Zhan Z. Salvia miltiorrhiza and Carthamus tinctorius extract prevents cardiac fibrosis and dysfunction after myocardial infarction by epigenetically inhibiting Smad3 expression. Evid Based Complement Alternat Med. (2019) 2019:6479136. doi: 10.1155/2019/6479136

32. Zhou X, Cheung CM, Yang JM, Or PM, Lee WY, Yeung JH. Danshen (Salvia miltiorrhiza) water extract inhibits paracetamol-induced toxicity in primary rat hepatocytes via reducing CYP2E1 activity and oxidative stress. J Pharm Pharmacol. (2015) 67:980-9. doi: 10.1111/jphp.12381

33. Wang F, He Q, Wang J, Yuan Q, Guo H, Chai L, et al. Neuroprotective effect of salvianolate lyophilized injection against cerebral ischemia in type 1 diabetic rats. BMC Complement Altern Med. (2017) 17:258. doi: 10.1186/s12906-017-1738-8

34. Beurel E, Grieco SF, Jope RS. Glycogen synthase kinase-3 (GSK3): regulation, actions, and diseases. Pharmacol Ther. (2015) 148:114-31. doi: 10.1016/j.pharmthera.2014.11.016

35. Wang Z, Ge Y, Bao H, Dworkin L, Peng A, Gong R. Redox-sensitive glycogen synthase kinase $3 \beta$-directed control of mitochondrial permeability transition: rheostatic regulation of acute kidney injury. Free Radic Biol Med. (2013) 65:849-58. doi: 10.1016/j.freeradbiomed.2013.08.169

36. Bao H, Ge Y, Peng A, Gong R. Fine-tuning of NFkappaB by glycogen synthase kinase $3 \beta$ directs the fate of glomerular podocytes upon injury. Kidney Int. (2015) 87:1176-90. doi: 10.1038/ki.2014.428
37. Gong R, Rifai A, Ge Y, Chen S, Dworkin LD. Hepatocyte growth factor suppresses proinflammatory NFkappaB activation through GSK3 $\beta$ inactivation in renal tubular epithelial cells. J Biol Chem. (2008) 283:740110. doi: 10.1074/jbc.M710396200

38. Sutherland C. What are the bona fide GSK3 substrates? Int J Alzheimers Dis. (2011) 2011:505607. doi: 10.4061/2011/505607

39. Jiang $\mathrm{Y}$, Bao $\mathrm{H}, \mathrm{Ge} \mathrm{Y}$, Tang $\mathrm{W}$, Cheng $\mathrm{D}$, Luo $\mathrm{K}$, et al. Therapeutic targeting of GSK3 $\beta$ enhances the Nrf2 antioxidant response and confers hepatic cytoprotection in hepatitis C. Gut. (2015) 64:168-79. doi: 10.1136/gutjnl-2013-306043

40. Zhou S, Wang P, Qiao Y, Ge Y, Wang Y, Quan S, et al. Genetic and pharmacologic targeting of glycogen synthase kinase $3 \beta$ reinforces the Nrf2 antioxidant defense against podocytopathy. J Am Soc Nephrol. (2016) 27:2289-308. doi: 10.1681/ASN.2015050565

41. Zhou Y, He W, Sun W, Zhou Z, Sun M, Xia P, et al. Sulfotanshinone IIA sodium ameliorates glucose peritoneal dialysis solutioninduced human peritoneal mesothelial cell injury via suppression of ASK1-P38-mediated oxidative stress. Cell Physiol Biochem. (2018) 46:2434-44. doi: 10.1159/000489650

42. Raisi A, Dezfoulian O, Davoodi F, Taheri S, Ghahremani SA. Salvia miltiorrhiza hydroalcoholic extract inhibits postoperative peritoneal adhesions in rats. BMC Complement Med Ther. (2021) 21:126. doi: 10.1186/s12906-021-03300-7

Conflict of Interest: The authors declare that the research was conducted in the absence of any commercial or financial relationships that could be construed as a potential conflict of interest.

Publisher's Note: All claims expressed in this article are solely those of the authors and do not necessarily represent those of their affiliated organizations, or those of the publisher, the editors and the reviewers. Any product that may be evaluated in this article, or claim that may be made by its manufacturer, is not guaranteed or endorsed by the publisher.

Copyright (C) 2022 Zhou, Yao, Lu, Li, Han and Wang. This is an open-access article distributed under the terms of the Creative Commons Attribution License (CC BY). The use, distribution or reproduction in other forums is permitted, provided the original author(s) and the copyright owner(s) are credited and that the original publication in this journal is cited, in accordance with accepted academic practice. No use, distribution or reproduction is permitted which does not comply with these terms. 